\section{rev Psi}

Revista de Psicología (UNLP)

https://revistas.unlp.edu.ar/revpsi

Artículo de investigación

\title{
Incidencia del uso de pantallas en niñas y niños menores de 2 años
}

Ofelia Rodríguez Sas ${ }^{1}$

\section{Correspondencia}

rodriguezsas@gmail.com

Filiaciones institucionales

${ }^{1}$ Universidad de Flores (Argentina)

\author{
Lorena Cynthia Estrada ${ }^{1}$
}

\section{Resumen}

Aunque no existe consenso sobre las repercusiones de la digitalización en la salud y bienestar de niñas y niños, algunos estudios revelan la necesidad del acompañamiento adulto para seleccionar contenidos y establecer límites. Los bebés necesitan explorar el entorno para desarrollar habilidades cognitivas, sensoriales y lingüísticas. Como nuestra capacidad de adaptación aumenta a medida que maduramos, cualquier alteración temprana en el desarrollo ejecutivo repercute a corto, medio y largo plazo. El uso inadecuado de las TIC incide negativamente en la neuroplasticidad cerebral, que se traducirá en escasa autoestima, bajo nivel cognitivo o dificultades conductuales, según edad y habilidades asociadas. También podrá alterar visión, sueño, peso, desarrollo, funciones ejecutivas y conducta. El compromiso adulto con el uso responsable de herramientas tecnológicas es fundamental para garantizar el óptimo desarrollo de niñas y niños nacidos y criados en la era digital.

\section{Palabras clave}

tiempo de pantalla | bebés | efectos adversos | teléfono móvil | neurodesarrollo

\section{Cómo citar}

Rodríguez Sas, O. y Estrada, L. C.

(2021). Incidencia del uso de pantallas en niñas y niños menores de 2 años. Revista de Psicología. HTTPS://DX.DOI. ORG/10.24215/2422572XEO86

\section{Proceso editorial}

Recibido

7 ago. 2020

Aceptado

11 feb. 2021

Editores

Nicolás Alessandroni, Ana Moreno-Núñez | Facultad de Psicología, Universidad Autónoma de Madrid (España)
ISSN

2422-572X

Licencia

Licencia de Cultura Libre CC-BY 4.0

(Compartir - Adaptar - Atribuir)

Entidad editora

RevPsi es una publicación de la

Facultad de Psicología (Universidad

Nacional de La Plata, Argentina) 


\section{Incidência do uso de telas em meninas e meninos menores de 2 anos}

\section{Resumo}

Não há consenso sobre o impacto da digitalização na saúde e no bem-estar de meninas e meninos, mas alguns estudos alertam para a necessidade de acompanhamento de adultos para selecionar conteúdo e estabelecer limites. Os bebês precisam explorar o ambiente para desenvolver habilidades cognitivas, sensoriais e de linguagem. Conforme nossa capacidade de se adaptar de maneira flexível às mudanças aumenta à medida que amadurecemos, qualquer alteração precoce no desenvolvimento executivo tem consequências a curto, médio e longo prazo. $\mathrm{O}$ uso inadequado das TIC tem incidência negativa na neuroplasticidade cerebral, que se traduz em baixa autoestima, baixo nível cognitivo ou dificuldades comportamentais, dependendo da idade e das habilidades associadas. Ele também pode alterar a visão, sono, peso corporal, desenvolvimento, funções executivas e comportamento. O compromisso dos adultos com o uso responsável de ferramentas tecnológicas é essencial para garantir o desenvolvimento ideal de meninas e meninos nascidos e criados na era digital.

\section{Palavras-chave}

tempo de tela | bebês | efeitos adversos | telefone celular | neurodesenvolvimento

\section{Incidence of screen time in children under 2 years old}

\section{Abstract}

There is no consensus about the consequences of early exposure to digital media on children's health and wellness, but some researchers warn about the need for adult supervision to screen out content and set up exposure rules. Babies need to explore the environment to develop new cognitive, sensory and language skills. Since our capacity to adapt swiftly to a changing environment develops as we grow up, early disturbance in the development of executive function may cause short-, mid-, and long-term consequences. Misuse of ICT has a negative impact on brain neuroplasticity, which will eventually lead to low self-esteem, poor cognitive performance or maladaptive behaviour, depending on age and related skills. It might also affect sight, sleep, weight, development, executive function and behaviour. Adults' commitment to responsible use of technology tools is essential to ensure the optimal development of children born and raised in digital times.

\section{Keywords}

screen times | babies | adverse effects | mobile phone | neurodevelopment 


\section{Aspectos destacados del trabajo}

- El exceso de pantallas tiene incidencia negativa en la neuroplasticidad cerebral de niñas y niños.

- También puede alterar visión, sueño, peso corporal, desarrollo, funciones ejecutivas, lenguaje, habilidades sociales y conducta.

- Es fundamental el acompañamiento antes de los 2 años, para seleccionar contenidos y establecer límites.

- Ni la función materna ni la función paterna pueden ser sustituidas por ningún dispositivo tecnológico.

El presente artículo tiene como objetivo analizar cuál es la incidencia de los hábitos y el tiempo de exposición a pantallas en niñas y niños de 0 a 2 años de edad, incluidos los diversos dispositivos tecnológicos utilizados universalmente por las familias en la actualidad. Si bien no existe un consenso unívoco sobre cuáles han de ser las repercusiones de la digitalización en la salud y el bienestar de niñas y niños, en los últimos años varios autores han demostrado que la exposición a pantallas es prácticamente universal, mientras que la adquisición de habilidades para utilizarlas se inicia cada vez más temprano (Waisman et al., 2018; Zimmerman et al., 2007). Existen razones tanto teóricas como empíricas para creer que es mayor la probabilidad de que los efectos de la exposición a los medios digitales sean más adversos antes de los 30 meses de edad aproximadamente (Zimmerman et al., 2007; Radesky y Christakis, 2016; Velasco Rust y Almeida Monge, 2018; Tomé, 2019; Hutton, 2020).

A los fines de este trabajo, se ha llevado a cabo una tarea de revisión sistemática cualitativa a partir de las pautas metodológicas consensuadas por autores tales como Aguilera Eguía (2014), Grijalva et al. (2019), Gómez-Luna et al. (2014) y Ortiz (2005). Se definió el problema, se buscó y se organizó la información más relevante y actualizada y se realizó un minucioso análisis de la misma, jerarquizando los estudios con una base científica más sólida, para luego elaborar conclusiones y recomendaciones.

\section{El uso de pantallas en la actualidad}

De acuerdo con la Real Academia Española (2019, definición 3), una pantalla electrónica consiste en una superficie sobre la cual se proyectan imágenes. Por lo tanto, a los fines de este trabajo, el concepto de pantalla se homologará a cualquier dispositivo digital que proyecte imágenes, como televisores, computadoras, tabletas, teléfonos móviles o celulares y consolas de videojuegos, entre otros.

Actualmente, el uso de dispositivos móviles se ha generalizado al interior de las familias, atravesando todas las escalas sociales y rangos etarios, en particular 
desde muy corta edad (Waisman et al., 2018; Zimmerman et al., 2007). Aunque la televisión es el medio más utilizado, las pantallas de dispositivos móviles también ocupan un tiempo considerable. Según un estudio llevado a cabo por Waisman et al. (2018) entre niñas y niños de 6 meses a 5 años, antes de los 2 años el 80,3\% de los participantes miraba televisión y el 37,4 \% utilizaba pantallas táctiles con asistencia. Aunque se registró una diferencia significativa respecto de la tenencia de aparatos electrónicos entre los hogares de mayores y menores ingresos, prácticamente todas las familias poseían televisores y teléfonos inteligentes (o smartphones). Otro hallazgo interesante de este estudio es que las madres que cuentan con estudios superiores refieren dedicarle mayor tiempo a la lectura. En una investigación realizada por Zimmerman et al. (2007), a los 3 meses de edad alrededor del $40 \%$ de los niños veía televisión, DVD o videos con regularidad, mientras que a los 24 meses dicho porcentaje ascendía al $90 \%$, siendo los 9 meses la edad media de iniciación en el uso de medios digitales. El tiempo promedio de exposición diaria era de 1 hora por día para los menores de 12 meses y de más de 1,5 horas a los 24 meses.

Si bien aún no se ha logrado clarificar de qué manera el uso indiscriminado de pantallas podría afectar tales procesos madurativos, se ha demostrado que la exposición temprana a la televisión se asocia con problemas de atención en los años siguientes (Christakis et al., 2004). Algunos investigadores (Li et al., 2020) han logrado asociar el uso excesivo de pantallas en niñas y niños con indicadores de salud física, conductual y psicosocial, mientras que otros han sistematizado los hallazgos de diversos estudios para discriminar cuáles son las variables que se correlacionan más específicamente con los hábitos de uso de los medios digitales durante la infancia (Duch et al., 2013). Ciertos estudios (Radesky y Christakis, 2016) incluso advierten sobre la necesidad de acompañamiento por parte de los adultos antes de los 2 años, a fin de seleccionar contenidos y establecer límites de exposición. Dicha dinámica debe incluir la posibilidad de explorar el mundo circundante para desarrollar las correspondientes habilidades cognitivas, sensoriales y lingüísticas (Waisman et al., 2018).

El cerebro tardará al menos 18 meses en desarrollarse para poder comprender que los símbolos de la pantalla tienen un equivalente en el mundo real (Hill, 2016). Tal como explican Waisman et al. (2018), durante los primeros años de vida tiene lugar la etapa de desarrollo sensoriomotor, durante la cual se encuentra limitada la comprensión del contenido bidimensional que ofrecen las pantallas. Antes de los 2 años de edad, aún no se ha alcanzado la madurez suficiente en términos de control atencional y pensamiento simbólico como para poder transferir los conocimientos adquiridos a través de una pantalla a su aplicación en la vida real, que se desarrolla en tres dimensiones. Asimismo, durante los 3 primeros años de vida, se produce un rápido desarrollo del cerebro y sus múltiples conexiones, el cual puede verse afectado por diversos efectos ambientales (Zimmerman et al., 2007).

En 2016, la American Academy of Pediatrics (Academia Estadounidense de 
Pediatría [AAP]), publicó una declaración donde destaca que el acompañamiento es un aspecto crucial, ya que las niñas y niños menores de 2 años necesitan desplegar la exploración práctica y la interacción social con sus cuidadores para desarrollar habilidades cognitivas, lingüísticas, motoras y socioemocionales. Debido a que sus habilidades simbólicas, mnésicas y atencionales aún no han madurado lo suficiente, no pueden aprender de los medios digitales tradicionales del mismo modo que mediante las interacciones personales. A partir de los 15 meses, pueden adquirir vocabulario a partir de las pantallas táctiles en estudios de laboratorio, pero luego tienen dificultades para transferir ese conocimiento al mundo tridimensional. El principal factor que facilita este aprendizaje con medios digitales consiste en que las madres y los padres observen con ellos el contenido y luego vuelvan a enseñarlo. Un estudio que analizó las interacciones de calidad en el uso de pantallas táctiles (Zack y Barr, 2016) demostró que los bebés no comprenden fácilmente la equivalencia funcional entre una imagen $2 \mathrm{D}$ y un objeto $3 \mathrm{D}$, a menos que cuenten con el acompañamiento de personas significativas. Este hallazgo destaca la importancia no solo de la interacción con los cuidadores, sino también de la participación del bebé en el proceso.

Inicialmente, la AAP había publicado una recomendación (2011) en la que se desaconsejaba la exposición de niñas y niños menores de 2 años a pantallas móviles. Sin embargo, más tarde publicó un nuevo parámetro (2016) en el que reducía la edad de inicio de exposición a los 18 meses (excepto videollamadas), con el reparo de elegir contenidos educativos de alta calidad y procurar el acompañamiento adulto hasta los 2 años, para luego limitar el tiempo de pantalla a 1 hora o menos por día. Los informes de Unicef (2018), por su parte, establecen que la obligación de proteger a niñas y niños dentro del mundo digital incluye no solo a familias y escuelas, sino también a gobiernos y otras instituciones.

\section{El rol parental}

Debido a la importancia de la interacción parental en el desarrollo infantil, la presencia o ausencia de la madre y/o el padre durante la exposición es de suma relevancia (Zimmerman et al., 2007). Para Potes y Filet Larrea (2018), cada sistema familiar forma parte de un contexto sociocultural que lo moldea y lo define, aunque las necesidades humanas continúan siendo las mismas y el desarrollo debe seguir su ritmo natural. Para poder crecer y desarrollarse en forma saludable, todas las niñas y niños necesitan saber que cuentan con una figura de cuidado y contención que está dispuesta a escuchar y acompañar. Ni la función materna ni la función paterna pueden ser sustituidas por ningún dispositivo tecnológico.

Cierto es que la exposición a pantallas de televisión o DVD en niñas y niños muy pequeños ha aumentado en términos de precocidad y frecuencia. Este fenómeno parece estar impulsado tanto por las creencias de las madres y los padres respecto de su valor educativo y recreativo como por la necesidad de delegar la tarea de cuidado 
(Zimmerman et al., 2007). Algunos informes (Rideout y Hamel, 2006; He et al., 2005; Zimmerman et al., 2007) han documentado diversos motivos utilizados por las familias para justificar el uso del televisor, entre los cuales se destacan su función como niñera electrónica y la creencia de que constituye una forma de entretenimiento. Además, muchas madres y padres creen que los videos y los programas televisivos para bebés ofrecen características educativas positivas (Garrison y Christakis, 2005; Linebarger y Walker, 2005), o bien consideran que los medios de comunicación constituyen una poderosa herramienta educativa que ofrece beneficios para el desarrollo intelectual (Rideout et al., 2003; Vandewater et al., 2007). Muchas niñas y niños (una quinta parte del rango entre 0 y 2 años; un tercio del rango entre 3 y 6 años) tienen un televisor en su dormitorio para que se liberen otros televisores de la casa y puedan ser utilizados por distintos miembros de la familia (Vandewater et al., 2007). En este sentido, se ha demostrado que lo que determina que las pantallas táctiles sean herramientas de enseñanza eficaces es la calidad de la interacción entre la madre y el bebé (Zack y Barr, 2016). Muchas madres y padres tienen una sensación de incompetencia acompañada por la fantasía de que serán las pantallas las encargadas de enseñarles a sus hijas e hijos mucho más de lo que ellas/os son capaces, para que puedan superar las falencias de su propia educación. Además, se preocupan ante la posibilidad de que pierdan oportunidades educativas si no se les habilita el uso de dispositivos tecnológicos (Rodríguez Sas y Estrada, 2019).

Según informa la AAP (2016), la mayoría de las opciones clasificadas como educativas en las tiendas de aplicaciones no cuentan con suficiente evidencia de eficacia ni se basan en planes de estudio estandarizados, a la vez que tampoco se sustentan en el trabajo realizado por especialistas en desarrollo y educación. Por eso, esta institución recomienda informarles a las familias que las habilidades cognitivas superiores y las funciones ejecutivas imprescindibles para lograr un buen desempeño escolar - tales como la perseverancia en las tareas, el control de los impulsos, la regulación emocional y el pensamiento creativo y flexible- se transmiten mejor mediante el juego no estructurado y social (no digital), así como mediante las interacciones empáticas entre madres/padres e hijas/os. En este sentido, se ha demostrado que la interacción cara a cara es imprescindible para preservar el desarrollo de las competencias sociales y emocionales en las niñas y niños pequeños (Detnakarintra et al., 2019).

Para Zimmerman et al. (2007), estos resultados tienen una gran relevancia en la práctica pediátrica y psicológica. Por un lado, la percepción de que la televisión podría ofrecer un potencial beneficio educativo puede utilizarse para reforzar el mensaje referido a la necesidad de que las niñas y niños estén acompañados por una persona adulta. De este modo, se podrían fomentar las interacciones que favorecen la adquisición de vocabulario durante la infancia. Por otro lado, se podría prestar especial atención e intervenir en aquellos hogares donde la exposición a la televisión es excesiva. Por último, las madres y los padres podrían recibir datos reales sobre la cantidad de tiempo que consumen televisión los pares de la misma edad, para poder tomar decisiones informadas que no solo se basen en sus propias percepciones. 


\section{Límites y reglas de uso}

Para Waisman et al. (2018), no se puede decir que las pantallas fijas y/o móviles sean buenas ni malas. En el grupo etario objeto de esta investigación, la diferencia radica en el modo en el que son utilizadas por las madres y los padres, la forma en la que introducen a sus hijas e hijos en la cultura tecnológica y la manera en la que los preservan de potenciales riesgos. Rodríguez Sas y Estrada (2019) señalan que a menudo las madres y los padres suelen expresar preocupación por sus propios hábitos de uso del teléfono móvil frente a sus hijas e hijos, pues este actúa como un distractor que interfiere en la interacción. Cuando el uso de los dispositivos móviles se torna excesivo por parte de los adultos, disminuye el intercambio verbal y no verbal en la familia, lo cual puede generar conflictos con los menores (Waisman et al., 2018).

Asimismo, cuando los adultos dejan el televisor encendido como sonido de fondo, este estímulo actúa como un distractor que impide las interacciones entre madres/padres e hijas/os, así como también el despliegue del juego (AAP, 2016). Del mismo modo, el uso intensivo de los dispositivos móviles por parte de los adultos se correlaciona con menos interacciones (verbales y no verbales) y un mayor nivel de conflicto con los menores, siendo un fuerte predictor de los futuros hábitos de uso de los medios digitales por parte de las niñas y niños. Esto significa que si las madres y los padres lograran reducir el uso de pantallas y a la vez mejorar la calidad de las interacciones con sus hijas e hijos, podrían generar un cambio de comportamiento significativo.

\section{Áreas comprometidas por el uso de pantallas}

Si bien los dispositivos tecnológicos generan un gran atractivo entre niñas y niños, las funciones digitales también pueden actuar como distractores del proceso de aprendizaje. Además, la exposición excesiva o inapropiada a pantallas (p. ej., contenido violento o adulto) trae aparejados otros riesgos para la salud, en particular en las áreas de sueño, obesidad, desarrollo infantil, funciones ejecutivas y conductas agresivas (Radesky y Christakis, 2016). Por su parte, Mendieta Pineda (2017) afirma que el tiempo de pantalla excesivo genera problemas emocionales y conductuales que afectan tanto el crecimiento como el desarrollo cognitivo de niñas y niños. Sobre la base de la evidencia científica recabada hasta el momento, los efectos no deseados de la exposición excesiva a pantallas pueden clasificarse en dos grandes grupos, según se manifiesten de inmediato en el desarrollo infantil o bien se vayan presentando en las etapas posteriores del crecimiento.

\section{Efectos tempranos en el neurodesarrollo}

Tal como explican García-Molina et al. (2009), durante los primeros años de vida emergen distintas capacidades cognitivas que luego constituirán las funciones ejecutivas, en las que se distinguen dos fases: durante la primera - que comprendería los 3 primeros años de vida- emergen las capacidades básicas que posteriormente 
permitirán un adecuado control ejecutivo; mientras que en la segunda tiene lugar un proceso de integración en el cual se coordinarían las capacidades básicas que emergieron previamente. Continúan los autores describiendo la evolución: en la segunda mitad del primer año, surgen formas simples de control inhibitorio; alrededor del segundo año, aumenta la capacidad de mantenimiento y manipulación de la información, junto con la inhibición de respuestas, para permitir un relativo control cognitivo sobre la conducta; antes del tercer año, ya han aparecido gran parte de las habilidades básicas necesarias para realizar tareas ejecutivas; entre el tercer y el quinto año, se desarrollan ampliamente las habilidades cognitivas que constituyen el núcleo de las funciones ejecutivas, que permiten mantener, manipular y transformar información para autorregular y adaptar la conducta a los cambios del entorno. Como el desarrollo de las funciones ejecutivas es de curso lento y progresivo, su espectro de vulnerabilidad es excepcionalmente amplio. Su normal devenir es crucial no solo para el funcionamiento cognitivo per se, sino también para el desarrollo social y afectivo de niñas y niños. Como nuestra capacidad para hacer frente a situaciones novedosas y adaptarnos con flexibilidad a los cambios aumenta a medida que maduramos, cualquier alteración temprana en el desarrollo ejecutivo limita esa capacidad con consecuencias a corto, medio y largo plazo.

Para Tomé (2019), la dependencia y el uso indebido de los teléfonos inteligentes tienen una incidencia negativa en la neuroplasticidad cerebral de niñas y niños, que en definitiva se hará evidente en forma de escasa autoestima, bajo nivel cognitivo o dificultades conductuales, según corresponda a su edad y habilidades asociadas. Señala el autor que, según estudios de anátomo-fisiología neurodesarrollante, entre la edad de 0 y 6 años el uso excesivo del teléfono móvil tiene una incidencia significativa en el desarrollo de la plasticidad neuronal, siendo este el periodo donde se presenta la mayor cantidad de modificaciones en la forma de los giros y surcos cerebrales, que en último término se traducen en forma intelectual, actitudinal y conductual. Un estudio reciente realizado a 47 niñas y niños en edad preescolar mediante la utilización de tecnología de imágenes (Hutton et al., 2020) encontró una correlación entre la exposición excesiva a pantallas según los estándares de la AAP (2016) y una menor organización microestructural y mielinización de la sustancia blanca del cerebro, que cumple una importante función en el desarrollo del lenguaje y las habilidades de alfabetización. El uso de pantallas también se asoció con puntuaciones más bajas en las mediciones conductuales segmentadas por edad.

La excesiva exposición a pantallas de niñas y niños también puede interrumpir las comunicaciones con sus cuidadores y su entorno, porque al estar concentrados frente a las pantallas desaprovechan oportunidades de interacciones sociales verbales y no verbales que resultan de vital importancia para estimular un crecimiento óptimo mediante la práctica de habilidades interpersonales, motoras y comunicacionales (Hernández, 2019). Debido a que antes de los 2 años el aparato psíquico aún no ha madurado y se encuentra en desarrollo, las niñas y niños son sumamente dependientes de la interacción con adultos para decodificar y significar los estímulos que reciben (Krynski et al., 2017). Si bien los bebés pueden fijar la mirada en los 
colores brillantes y el movimiento de la pantalla, sus cerebros no son capaces de discernir o significar todas esas extrañas imágenes.

Un estudio reciente demostró la existencia de una asociación significativa entre el uso de dispositivos móviles y un retraso del lenguaje expresivo según lo informado por familias de niñas y niños de 18 meses de edad (van den Heuvel et al., 2019). En el caso particular de la televisión, reduce las situaciones de interacción y juego entre madres/padres e hijas/hijos, que son cruciales para el desarrollo temprano del lenguaje (Hernández, 2019). Tal como señala Hill (2016), basta con dejar el televisor encendido con el sonido de fondo, aun cuando nadie se encuentre frente a la pantalla, para que pueda generar un efecto de retraso en el desarrollo del lenguaje. Normalmente, un/a cuidador/a podría pronunciar unas 940 palabras por hora mientras comparte tiempo con una niña o niño, pero si la televisión se encuentra encendida, esa cantidad se reduce a 770 , con la consecuente merma en el aprendizaje. Cabe destacar que según estudios específicos (Rideout et al., 2003) dos de cada tres niños de 0 a 6 años viven en hogares donde el televisor se deja encendido al menos la mitad del tiempo. Tal como señalan Eskritt et al. (2008), las niñas y niños aprenden palabras dentro de un contexto social donde sus cuidadores ofrecen respuestas e incentivos rápidos a su intención comunicativa. Cuando los adultos no participan de los intercambios propuestos por sus hijas/os, rompen una premisa pragmática importante de la comunicación, que ya se puede detectar a los 3 años de edad. Además, cuando los adultos se encuentran ensimismados en sus propias pantallas, disminuye la interacción verbal entre ellos, lo cual reduce las posibilidades de que sus hijas e hijos puedan escuchar incidentalmente sus diálogos (Radesky et al., 2015).

Algunas investigaciones (Velasco Rust y Almeida Monge, 2018) demuestran la existencia de una relación entre el tiempo de exposición a pantallas y el desarrollo del coeficiente intelectual (CI) en niñas y niños entre 12 y 48 meses de edad, considerando que el $42 \%$ de los casos estudiados evidencian un desarrollo deficiente del CI cuando el inicio de la exposición se ubica entre los 6 meses y los 2 años. El factor determinante de este resultado sería la disminución de la interacción familiar a causa de la utilización de dispositivos tecnológicos por parte de niñas/os y cuidadores. Además, una edad de inicio más temprana y una mayor cantidad de horas de exposición son predictores significativos independientes de un déficit en el funcionamiento ejecutivo de niñas y niños preescolares (AAP, 2016). Otros autores señalan que la exposición a la televisión antes de los 3 años de edad podría tener efectos adversos moderados sobre el desarrollo cognitivo posterior (Zimmerman y Christakis, 2005). También se han demostrado efectos adversos sostenidos en los niveles de atención, el desarrollo del lenguaje, las habilidades de lectura y la memoria a corto plazo, cuando la exposición a pantallas se produce antes de los 18 meses (Hill, 2016).

Además, el uso excesivo de dispositivos tecnológicos produce alteraciones vinculares (Krynski et al., 2017). Cuando las niñas y niños pequeños se exponen a una pantalla, pierden la oportunidad de ensayar y adquirir habilidades interpersonales, motoras y comunicacionales (Madigan et al., 2019). Además de ver los rostros y oír las voces 
de sus cuidadores, en la primera etapa de la vida es preciso tocar, sacudir y tirar los objetos. Si bien las aplicaciones pueden enseñar a presionar, deslizar y mover los dedos por la pantalla, las investigaciones demuestran que estas destrezas no se homologan al aprendizaje en el mundo real (Hill, 2016). Por eso, es importante que la tecnología no sustituya las actividades sociales ni el juego al aire libre (Krynski et al., 2017).

\section{Efectos posteriores durante el desarrollo}

Existe evidencia científica sobre los efectos negativos (reversibles e irreversibles) de la sobreexposición a la luz que emiten las pantallas de los dispositivos electrónicos retroiluminados y los síntomas asociados al uso de estos dispositivos. En particular, se registra un aumento de casos de conjuntivitis, blefaritis, queratitis, cataratas y retinopatías entre las poblaciones de mayor riesgo (niñas y niños, jóvenes, personas especialmente sensibles y adultos mayores) (Roda et al., 2015).

Asimismo, el efecto lumínico de las pantallas produce trastornos del sueño (Krynski et al., 2017; Hill, 2016; AAP, 2016), en particular después de las ocho de la noche, ya que inhibe la secreción de melatonina (Garrison et al., 2011) y dificulta la conciliación del sueño, lo cual puede producir trastornos emocionales y de concentración a largo plazo (Mendieta Pineda, 2017). En efecto, un metaanálisis llevado a cabo recientemente ( $\mathrm{Li}$ et al., 2020) indicó que el tiempo excesivo frente a una pantalla se asocia con una menor duración del sueño en la primera infancia. Este hallazgo ratifica los resultados de un estudio realizado en el Reino Unido a más de 700 bebés de 6 a 36 meses (Cheung et al., 2017), que revelaron una asociación significativa entre la frecuencia de uso de la pantalla táctil y la cantidad de sueño, así como un inicio del sueño más prolongado. Estas alteraciones podrían deberse a que la luz azul brillante de las pantallas es interpretada por el cerebro como la luz del día, lo cual afecta el ritmo circadiano y los niveles de excitación, que también podrían verse aumentados por el contenido visualizado. No se encontró ninguna correlación con la cantidad de despertares nocturnos. También el aumento de la exposición a la televisión de fondo se asoció en forma independiente con una reducción del sueño diurno. Asimismo, la duración del sueño nocturno y el inicio del sueño se correlacionaron negativamente con la duración de la siesta durante el día, que puede verse afectada por una reducción en la calidad de aquel, o viceversa. Uno de los mecanismos que explicaría esta correlación es la luz azul emitida por las pantallas de los dispositivos digitales, que suprime los niveles de melatonina y en consecuencia modifica el ritmo circadiano. Otras hipótesis señalan la sustitución de tiempo de sueño por tiempo de pantalla, el aumento del nivel de activación física y psíquica a causa de la exposición, o bien el aumento del nivel de alerta nocturna y la reducción del tiempo de sueño REM debido al uso de medios digitales. Algunos investigadores (Beyens y Nathanson, 2019) también han demostrado que la duración del sueño se va reduciendo a medida que aumenta la edad, ya que se va reemplazando tiempo de sueño por tiempo de uso de los medios digitales. Sin embargo, consideran más importante limitar el tiempo de exposición a pantallas en niñas y niños que en adolescentes. Otro hallazgo relevante de estos autores es que las pantallas de menor tamaño (p. ej. teléfonos móviles) tenía 
un mayor impacto en la reducción del sueño que las de aquellos dispositivos de mayores dimensiones (como los televisores o las tabletas).

De la evidencia científica (Li et al., 2020) surge también que el tiempo excesivo frente a pantallas no solo se asocia con una menor duración del sueño, sino también con la presencia de sobrepeso, obesidad y sedentarismo (Krynski et al., 2017) en niñas y niños de edad preescolar. Se ha demostrado que el índice de masa corporal (IMC) aumentaba por cada hora semanal de consumo de medios digitales (AAP, 2016). También se ha comprobado que la presencia de un televisor en una habitación infantil es un marcador de mayor riesgo de tener sobrepeso (Dennison et al., 2002). Asimismo, la exposición a pantallas que no va acompañada de un componente interactivo o físico fomenta el sedentarismo e impide practicar habilidades de motricidad gruesa como caminar y correr, lo cual demora el desarrollo motor (Madigan et al., 2019). La revisión sistemática de diversos estudios sobre la relación entre el tiempo de pantalla y los indicadores de salud en bebés y preescolares reveló que una mayor exposición a medios digitales aumentaba el riesgo de padecer adiposidad, problemas de sueño, comportamiento agresivo, dolor musculoesquelético, acoso escolar en los años siguientes, hábitos alimentarios menos saludables, descenso en las funciones ejecutivas, el desarrollo motor y el nivel de actividad física, sedentarismo y dificultades conductuales y emocionales (Li et al., 2020).

Los investigadores también admiten que el uso excesivo de dispositivos tecnológicos puede favorecer la aparición de depresión y ansiedad infantil (Unicef, 2018). Una investigación realizada por la Universidad de Calgary (Madigan et al., 2019) reveló que el tiempo de exposición a pantallas puede afectar la capacidad de niñas y niños para desarrollarse de manera óptima, al demostrar que cuanto mayor había sido el tiempo de exposición a pantallas entre los 2 y 3 años de edad, peor fue el desempeño en una prueba de desarrollo que evaluaba comunicación, habilidades motoras gruesas y finas, resolución de problemas y habilidades sociales.

Una de las principales investigaciones realizada en Canadá con una muestra de más de tres mil participantes (Tamana et al., 2019) analizó la relación entre el tiempo de exposición a pantallas y los problemas de conducta en niñas y niños preescolares. Los resultados demostraron que al superar el umbral de dos horas diarias de exposición aumentaba el riesgo de aparición de morbilidad clínica, en particular el déficit atencional, con un riesgo 7,7 veces mayor de cumplir con los criterios de TDAH. Esta correlación fue la más significativa en comparación con otros factores de riesgo tales como duración del sueño, estrés parental y variables socioeconómicas. Al mismo tiempo, las niñas y niños que realizaban más de dos horas semanales de actividad física organizada tenían menos probabilidades de desarrollar problemas relacionados con la salud mental.

\section{Conclusiones y recomendaciones}

Uno de los estímulos fundamentales para impulsar el desarrollo infantil es la interacción significativa de las niñas y niños con sus cuidadores, sin que medie 
el efecto distractor de las pantallas. A su vez, la excesiva exposición a pantallas se asocia con efectos adversos tales como retrasos cognitivos y peor desempeño escolar. La evidencia científica ha demostrado que el tiempo de exposición a pantallas se correlaciona con resultados más bajos en las evaluaciones de desarrollo durante los primeros años de vida. Por ello, el compromiso de las familias con el uso responsable de las herramientas tecnológicas es fundamental para garantizar el óptimo desarrollo de las niñas y niños nacidos y criados en la era digital.

Si bien la declaración de políticas de la AAP (2016) recomienda no exponer a menores de 18 meses al uso de dispositivos digitales, así como acompañar su uso entre los 18 y 24 meses, las investigaciones analizadas en este estudio han aportado suficiente evidencia científica sobre los potenciales efectos adversos de la excesiva exposición a pantallas antes de los 2 años de edad. Por lo tanto, se requiere la intervención activa de madres y padres para evitar hábitos de uso de dispositivos tecnológicos potencialmente nocivos, reemplazando el tiempo sedentario frente a la pantalla por juegos enérgicos al aire libre y a la vez mantener una adecuada higiene del sueño (Tremblay et al., 2017). Asimismo, se deberá hacer foco en las siguientes recomendaciones (AAP, 2016):

- No usar pantallas antes de ir a dormir, durante la alimentación o como instrumento de calma.

- No exponer a niñas y niños a contenidos violentos o inapropiados para su edad.

- Limitar el tiempo de exposición a pantallas de acuerdo con las recomendaciones de la AAP.

- Establecer reglas de uso de la tecnología para toda la familia.

- No ofrecer modelos de consumo excesivo de tecnología; enseñar con el ejemplo conductas de uso responsable y saludable, dado que la imitación es el principal factor de influencia para el uso de pantallas a esta edad.

- Compartir el tiempo de pantalla con sus hijas e hijos, en lugar de usar los dispositivos tecnológicos como sustitutos de cuidadores.

Por su parte, será también responsabilidad de instituciones sociales, políticas y gubernamentales velar por el cuidado de las niñas y niños en el mundo digital, mediante la implementación de normativas, recomendaciones, programas de difusión y concientización y cualquier otra medida pertinente basada en los avances científicos dentro de este campo de estudio.

Resulta curioso observar que la RAE (2019, definición 5) también incluye una acepción que define la pantalla como algo o alguien que se coloca delante de un objeto o una persona para ocultarla o hacerle sombra. En este sentido, Ubieto y Pérez Álvarez (2018) ponen el acento en recuperar los vínculos cara a cara en tiempo 
real, como la conversación y el juego, en contraposición con el otro digital que se sustenta en una relación sujeto-objeto no mediatizada por un Otro. Al propiciar este modo de vinculación con los otros, también se incentiva la relación consigo mismo, para aumentar la tolerancia al aburrimiento en el proceso de construcción subjetiva. Las niñas y niños tienen derecho a explorar soluciones mediante prueba y error y a transitar a su tiempo el camino hacia la adultez. Mientras que las personas adultas debemos aprender por nuestra parte a tolerar la espera y la incertidumbre que supone brindarles nuestro acompañamiento a lo largo de su desarrollo.

El objeto de estudio de este trabajo cobra además una particular relevancia dado el actual contexto de crisis sanitaria y social ocasionada por la pandemia de COVID-19. Debido a la necesidad de mantener el aislamiento social, muchas familias con niñas y niños pequeños transcurren muchas horas dentro del hogar, lo cual aumenta de manera exponencial el riesgo de exposición a estímulos audiovisuales (Pinchak, 2020). Por tal motivo, se recomienda conservar los horarios habituales y los períodos normales de sueño-vigilia del modo más cercano a la rutina cotidiana anterior al inicio del brote (Romero et al., 2020). También es importante para estos autores programar actividades diversas y alternadas para evitar la sobreexposición a pantallas. Para aprovechar al máximo el tiempo de confinamiento, es importante estimular la creatividad mediante tareas artísticas y lúdicas que permitan afianzar los vínculos. A fin de fortalecer la salud emocional de niñas y niños, los adultos deben modelar una autopercepción positiva y una adecuada regulación de las emociones. Mientras que para promover la salud digital, es preciso brindarles acompañamiento en su tránsito por el mundo cibernético.

\section{Futuras líneas de investigación}

La información recabada y analizada en el presente trabajo pone de relieve la necesidad de realizar más estudios que permitan identificar cuáles son las implicancias de la exposición temprana a pantallas para el neurodesarrollo, en particular durante las etapas de crecimiento rápido del cerebro en la primera infancia. Sería relevante para las futuras investigaciones poner el foco en detectar la existencia de correlaciones por franja etaria, por tipo de medio utilizado y/o por características del contenido expuesto, a fin de optimizar las intervenciones pertinentes y las políticas públicas de difusión, educación y asistencia. Asimismo, será preciso llevar a cabo estudios más profundos y focalizados para evaluar y ponderar el impacto específico del actual contexto epidemiológico en los hábitos de uso de medios digitales. 


\section{Referencias}

AAP, American Academy of Pediatrics Council on Communications and Media (2011). Media use by children younger than 2 years. Pediatrics, 128(5), 1040-4045. HTTPS://DOI.ORG/10.1542/ PEDS.2011-1753

AAP, American Academy of Pediatrics Council on Communications and Media (2016). Media and young minds. Pediatrics, 138(5), e20162591. HTTPS://DOI.ORG/10.1542/PEDS.2016-2591

Aguilera Eguía, R. (2014). ¿Revisión sistemática, revisión narrativa o metaanálisis? Revista de la Sociedad Española del Dolor, 21(6), 359-360. HTTP://DX.DOI.ORG/10.4321/S1134$\underline{80462014000600010}$

Beyens, I. y Nathanson, A. I. (2019). Electronic media use and sleep among preschoolers: Evidence for time-shifted and less consolidated sleep. Health Communication, 34(5), 537-544. HTTPS://DOI.OR G/10.1080/10410236.2017.1422102

Christakis, D. A., Zimmerman, F. J., Di Giuseppe, D. L. y McCarty, C. A. (2004). Early television exposure and subsequent attentional problems in children. Pediatrics, 113(4), 708-13. HTTPS:// DOI.ORG/10.1542/PEDS.113.4.708

Cheung, C. H., Bedford, R., De Urabain, I. R. S., Karmiloff-Smith, A., y Smith, T. J. (2017). Daily touchscreen use in infants and toddlers is associated with reduced sleep and delayed sleep onset. Scientific Reports, 7, 46104. HTTPS://DOI. ORG/10.1038/SREP46104

Dennison, B. A., Erb, T. A. y Jenkins, P. L. (2002). Television viewing and television in bedroom associated with overweight risk among low-income preschool children. Pediatrics, 109(6), 1028-1035. HTTPS://PEDIATRICS. AAPPUBLICATIONS.ORG/CONTENT/109/6/1028. SHORT

Detnakarintra, K., Trairatvorakul, P. Pruksananonda, C. y Chonchaiya, W. (2019). Positive mother-child interactions and parenting styles were associated with lower screen time in early childhood. Acta Paediatrica, 109(4). HTTPS://DOI.ORG/10.1111/APA.15007

Duch, H., Fisher, E. M., Ensari, I. y Harrington, A. (2013). Screen time use in children under 3 years old: A systematic review of correlates. International Journal of Behavioral Nutrition and Physical Activity, 10(102). HTTPs://DOI. ORG/10.1186/1479-5868-10-102
Eskritt, M., Whalen, J. y Lee, K. (2008). Preschoolers can recognize violations of the Gricean maxims. British Journal of Developmental Psychology, 26(3), 435-443. HTTPs://DOI. ORG/10.1348/026151007X253260

García-Molina, A., Enseñat-Cantallops, A., TirapuUstárroz, J. y Roig-Rovira, T. (2009). Maduración de la corteza prefrontal y desarrollo de las funciones ejecutivas durante los primeros cinco años de vida. Revista de Neurología, 48(8), 435440. HTTPS://DOI.ORG/10.33588/RN.4808.2008265

Garrison, M. M., y Christakis, D. A. (2005). A teacher in the living room?: Educational media for babies, toddlers and preschoolers: A background report prepared for Kaiser Family Foundation. Henry J. Kaiser Family Foundation. HTTPs://WwW. SCIRP.ORG/(S(LZ5MQP453EDSNP55RRGJCT55))/ REFERENCE/REFER EN CES PA P ERS. ASPX?REFERENCEID $=866217$

Garrison, M. M., Liekweg, K. y Christakis, D. A (2011). Media use and child sleep: The impact of content, timing, and environment. Pediatrics, 128(1), 29-35. HTTPS://DOI.ORG/10.1542/ PEDS.2010-3304

Gómez-Luna, E., Fernando-Navas, D., AponteMayor, G. y Betancourt-Buitrago, L. A. (2014). Metodología para la revisión bibliográfica y la gestión de información de temas científicos, a través de su estructuración y sistematización. Dyna, 81(184), 158-163. HTTPs://DOI. ORG/10.15446/DYNA.V81N184.37066

Grijalva, P. K., Cornejo, G. E., Gómez, R. R., Real, K. P. y Fernández, A. (2019). Herramientas colaborativas para revisiones sistemáticas. Revista Espacios, 40(25). HTTP://WWW. REVISTAESPACIOS.COM/A19V4ON25/19402509. HTML

He, M., Irwin J. D., Sangster Bouck L. M., Tucker P. y Pollett G. L. (2005). Screen-viewing behaviors among preschoolers parents' perceptions. American Journal of Preventive Medicine, $29(2), \quad 120-5 . \quad$ HTTPS://DOI.ORG/10.1016/J. AMEPRE.2005.04.004

Hernández, A. Q. (2019). Las pantallas y el desarrollo de los niños pequeños. HTTPS://WWW. CUBAHORA.CU/BLOGS/CONSULTAS-MEDICAS/ LAS-PANTALLAS-Y-EL-DESARROLLO-DE-LOSNINOS-PEQUENOS?PAGE $=3$ 
Hill, D. L. (28 de octubre de 2016). Por qué evitar que los bebés y los niños pequeños vean la televisión. Healthychildren.org. HTTPS://WWW. HEALTHYCHILDREN.ORG/SPANISH/FAMILY-LIFE/ MEdia/PAGINAS/WHY-TO-AVOID-TV-BEFOREAGE-2.ASPX

Hutton, J. S., Dudley, J., Horowitz-Kraus, T., DeWitt, T. y Holland, S. K. (2020). Associations between screen-based media use and brain white matter integrity in preschool-aged children. JAMA Pediatrics, 174(1), e193869-e193869. HTTPs://DOI. ORG/10.1001/JAMAPEDIATRICS.2019.3869

Krynski, L., Ciancaglini, A. y Goldfarb, G. (2017) Bebés, niños, adolescentes y pantallas: ¿qué hay de nuevo? Archivos Argentinos de Pediatría, 115(4), 404-408. HTTPS://SCP.COM.CO/WPCONTENT/UPLOADS/2014/13/BNAP.PDF

Li, C., Cheng, G., Sha, T., Cheng, W. y Yan, Y (2020). The relationships between screen use and health indicators among infants, toddlers, and preschoolers: A meta-analysis and systematic review. International Journal of Environmental Research and Public Health, 17(19), 7324. HTTPS://DOI.ORG/10.3390/IJERPH17197324

Linebarger, D. L. y Walker, D. (2005). Infants and toddlers' television viewing and language outcomes. American Behavioral Scientist, 48(5), 624-645. HTTPS://DOI. ORG/10.1177\%2F0002764204271505

Madigan, S., Browne, D., Racine, N., Mori, C y Tough, S. (2019). Association between screen time and children's performance on a developmental screening test. JAMA Pediatrics, 173(3), 244-250. HTTPS://DOI.ORG/10.1001/ IAMAPEDIATRICS.2018.5056

Mendieta Pineda, Z. L. (2017). Efectos adversos en el desarrollo visual y cognitivo en niños menores de 3 años relacionados con el tiempo excesivo de uso de pantallas digitales. Tesis de grado. Facultad de Ciencias de la Salud. Optometría. Universidad de La Salle. HTtps://CIENCIA.LASALLE.EDU.Co/ OPTOMETRIA/9

Ortiz, Z. (2005). ¿Qué son las revisiones sistemáticas? CIE. Academia Nacional de Medicina, Buenos Aires. HTtP://wWW.epidemiologia.ANM.edu AR/WP-CONTENT/UPLOADS/2018/06/QUE-SONLAS-REVISIONES-SISTEMATICAS- -2005.PDF

Pinchak, C. (2020). Pandemia por coronavirus (COVID-19); sorpresa, miedo y el buen manejo de la incertidumbre en la familia. Archivos de Pediatría del Uruguay, 91(2), 76-77. нттр:// DX.DOI.ORG/10.31134/AP.91.2.1
Potes, M. V. y Filet Larrea, G. J. (2018). Tecnofamilias: crecer en cavernas digitales. Eureka, 15(2). HTTPS://PSICOEUREKA.COM.PY/SITES/DEFAULT/ FILES/ARTICULOS/EUREKA-15-2-16.PDF

Radesky, J. S. y Christakis, D. A. (2016). Increased screen time: Implications for early childhood development and behavior. Pediatric Clinics of North America, 63(5), 827-839. HTTPs://DOI. ORG/10.1016/T.PCL.2016.06.006

Radesky, J., Miller, A., Rosenblum, K. L., Appugliese, D., Kaciroti, N. y Lumeng, J. C. (2015). Maternal mobile device use during a structured parentchild interaction task. Academic Pediatrics, 15(2), 238-244. HTTPS://DOI.ORG/10.1016/J. ACAP.2014.10.001

Real Academia Española (2019). Diccionario de la Lengua Española (23a ed.).

Rideout, V. J. y Hamel, E. (2006). The media family: Electronic media in the lives of infants, toddlers, preschoolers and their parents. The Henry J. Kaiser Family Foundation. HTTPs://WwW.KFF. ORG/WP-CONTENT/UPLOADS/2013/01/7500.PDF

Rideout, V. J., Vandewater, E. A. y Wartella, E. A. (2003). Zero to six: Electronic media in the lives of infants, toddlers and preschoolers. The Henry J. Kaiser Family Foundation. HTTPS://ERIC. ED.GOV/?ID $=$ ED 482302

Roda, C. S. R., Arias, C. B., Carrasco, M. J. P., Vila-Coro, V. A., Rojo, M. G., Navarro, C. B., y Gutiérrez, E. C. (2015). Síndrome de visión del ordenador (CVS): un nuevo reto para la prevención. Seguridad y Promoción de la Salud, 25(140). HTTPS://APP.MAPFRE.COM/ FUNDACION/HTML/REVISTAS/SEGURIDAD/N140/ ES/ARTICULO3.HTML

Rodríguez Sas, O. y Estrada, L. C. (2019). Comunicación familiar en la era del vacío: nuevas patologías asociadas al uso problemático del móvil. Revista de Psicología, 18(2), 105-123. HTTPS://DOI.ORG/10.24215/2422572XE040

Romero, C. C., del Mazo Fuente, A., Besada, M. D. y Hernández, M. D. L. O. R. (2020). Algunas aportaciones de la orientación educativa en red durante el confinamiento por la pandemia de COVID-19. Revista AOSMA, 28, 33-49. 